\title{
Accuracy of Oxygen Flow Delivered by Compressed-Gas Cylinders in Hospital and Prehospital Emergency Care
}

\author{
Frédéric Duprez PT MSc, Jean Bernard Michotte PhD PT, Gregory Cuvelier MSc PT, \\ Alexandre Legrand PhD MD, Sharam Mashayekhi MD, and Gregory Reychler PhD PT
}

\begin{abstract}
BACKGROUND: Oxygen cylinders are widely used both in hospital and prehospital care. Excessive or inappropriate $\mathrm{F}_{\mathrm{IO}}$, may be critical for patients with hypercapnia or hypoxia. Moreover, over-oxygenation could be deleterious in ischemic disorders. Supplemental oxygen from oxygen cylinder should therefore be delivered accurately. The aim of this study was to assess the accuracy of oxygen flows for oxygen cylinder in hospital and prehospital care. METHODS: A prospective trial was conducted to evaluate accuracy of delivered oxygen flows $(2,4,6,9$ and $12 \mathrm{~L} / \mathrm{min})$ for different oxygen cylinder ready for use in different hospital departments. Delivered flows were analyzed randomly using a calibrated thermal mass flow meter. Two types of oxygen cylinder were evaluated: 78 oxygen cylinder with a single-stage regulator and 70 oxygen cylinder with a dual-stage regulator. Delivered flows were compared to the required oxygen flow. The residual pressure value for each oxygen cylinder was considered. A coefficient of variation was calculated to compare the variability of the delivered flow between the two types of oxygen cylinder. RESULTS: The median values of delivered flows were all $\geq 100 \%$ of the required flow for single stage (range 100-109\%) and $<100 \%$ of required flow for dual stage (range 95-97\%). The median values of the delivered flow differed between single and dual stage. It was found that single stage is significantly higher than dual stage $(P=.01)$. At low flow, the dispersion of the measures for single stage was higher than with a high oxygen flow. Delivered flow differences were also found between low and high residual pressures, but only with single stage $(P=.02)$. The residual pressure for both oxygen cylinders (no. $=148$ ) ranged from 73 to 2,900 pounds per square inch, and no significant difference was observed between the 2 types $(P=.86)$. The calculated coefficient of variation ranged from $7 \%( \pm 1 \%)$ for dual stage to $8 \%( \pm 2 \%)$ for single stage. CONCLUSIONS: This study shows good accuracy of oxygen flow delivered via oxygen cylinders. This accuracy was higher with dual stage. Single stage was also accurate, however, at low flow this accuracy is slightly less. Moreover, with single stage, when residual pressure decreases, the median value of delivered flow decreased. Key words: oxygen gas cylinders; oxygen therapy; accuracy; hyperoxia; hypoxia. [Respir Care 2018;63(3):332-338. (c) 2018 Daedalus Enterprises]
\end{abstract}

\section{Introduction}

Oxygen therapy is widely used in both hospital and prehospital care. ${ }^{1-3}$ In 2008 , a study showed that, in the

Mr Duprez is affiliated with Service des Soins Intensifs, Hôpital Epicura, Site Hornu, Belgium; Institut de Recherche Expérimentale et Clinique (IREC), Pôle de Pneumologie, ORL \& Dermatologie, Service de Pneumologie, Université Catholique de Louvain, Brussels, Belgium; and Laboratoire de l'Effort et du Mouvement, Condorcet, Tournai, Belgium. Dr Mashayekhi is affiliated with Service des Soins Intensifs, Hôpital Epicura, Site Hornu, Belgium. Dr Michotte is affiliated with Western Switzerland University of Applied Sciences-Haute Ecole de Santé Vaud (HESAV), Filière physiothérapie, Lausanne, Switzerland. Dr Reychler is
United Kingdom, 15-17\% of hospitalized patients received oxygen at some point compared to $34 \%$ of patients who had been transported by ambulance. ${ }^{4}$

In these situations, oxygen therapy is delivered with wallmounted Thorpe tube or oxygen cylinders. Thorpe tubes are

affiliated with Institut de Recherche Expérimentale et Clinique (IREC), Pôle de Pneumologie, ORL \& Dermatologie, Service de Pneumologie, Université Catholique de Louvain, Brussels, Belgium. Mr Cuvelier is affiliated with Laboratoire de l'Effort et du Mouvement, Condorcet, Tournai, Belgium. Dr Legrand is affiliated with Service de Physiologie, Physiopathologie et Réadaptation Respiratoire, Université de Mons, Belgium.

The authors have disclosed no conflicts of interest. 


\section{Accuracy of Flow Delivered by Oxygen Cylinders}

used in hospitals to deliver an oxygen flow. A driving pressure is applied to the inlet of the Thorpe tube, and a float indicator rises in the tapered tube until the required flow is read. ${ }^{5}$ Oxygen cylinders are in steel or aluminum tanks containing the gas under high pressure. The high pressure must be decreased to administer oxygen safely to a patient. To decrease this pressure to an intermediate level - from 2,900 pounds per square inch (psi) to $44 \mathrm{psi}$ - oxygen cylinders are equipped with a pressure regulator. Two models of regulators exist: preset single-stage regulators, using a single inlet valve, and preset dual-stage regulators, using a series of valves. Working at this intermediate pressure, a flow meter regulates the outflow using variable-flow orifice restrictors. This simple technique uses calibrated ports to deliver predetermined flow. ${ }^{5}$ In Europe, the accuracy of flow measurement devices for medical gases is governed by the ISO 15002 standards. ${ }^{6}$ According to this standard, the delivered flow should not deviate from the required flow by $>0.5 \mathrm{~L} / \mathrm{min}$ when the required flow is $<5 \mathrm{~L} / \mathrm{min}$, and by $>10 \%$ above this threshold. In North America, the requirement standards are set by the Compressed Gas Association. These standards specify an allowable error of $10 \%$ above or below the required flow. ${ }^{5}$ In North America, gas pressure in oxygen cylinders is usually expressed in psi, while in Europe the unit is the bar $\left(\mathrm{kgf} / \mathrm{cm}^{2}\right) .{ }^{5}$ Oxygen cylinders and Thorpe tube flowmeters are routinely used successively, as in during intrahospital or prehospital transfers. Therefore, it is important to know whether these systems deliver similar levels of oxygen flow to maintain the same level of oxygenation.

In 2013, a study highlighted that several thousand deaths could be avoided each year in United Kingdom with controlled oxygen use. ${ }^{3}$ The accuracy of oxygen flow is therefore a key. Recent studies have examined the accuracy of Thorpe tubes in clinical situations. ${ }^{7,8}$ They observed that the required flow was different from the delivered flow using Thorpe tube. This difference can lead to over- or under-oxygenation of patients, which can be deleterious in various conditions, such as COPD, ischemic disorders, and for premature infants. However, few data exist regarding the use of oxygen cylinders with a flow meter. The aim of this study was to evaluate the accuracy of flow meters attached to oxygen cylinders that are ready to be used.

\section{Methods}

Flows delivered by flow meters were evaluated on successive oxygen cylinders ready for use, selected from two hos-

\footnotetext{
Correspondence: Gregory Reychler PhD PT, Service de Pneumologie, Cliniques Universitaires St-Luc (UCL), Brussels, Belgium. E-mail: gregory.reychler@uclouvain.be.
}

DOI: $10.4187 /$ respcare. 05657

\section{QUICK LOOK}

\section{Current knowledge}

Oxygen therapy is used for treating hypoxia. Oxygen gas cylinders can be used for delivering oxygen flow in pre- and intrahospital care. Supplemental oxygen delivered by oxygen cylinders is valuable in many clinical situations. The accuracy of oxygen flow meters from cylinders is essential to prevent over- or under-oxygenation.

\section{What this paper contributes to our knowledge}

This study shows good accuracy of oxygen flows delivered by oxygen gas cylinders in hospital and during prehospital care. However, the results report a statistical but limited difference between the investigated types of oxygen-gas cylinders. Moreover, the results show that inaccuracies may appear at low oxygen flow and/or low residual pressures in some types of cylinders. Clinicians should be aware of these differences when administering oxygen.

pital emergency departments, two ambulance services, and a firefighting brigade, in the Walloon region of Belgium, from March to May 2016. The oxygen cylinders analyzed were from the stock available on the days that the measures were taken.

\section{Measurements}

Gas Pressure. Before performing the flow measurements, the residual pressure of each oxygen cylinder was checked by direct reading on the manometer. Any gas pressure in oxygen cylinder $>1,450 \mathrm{psi}$ was considered as high pressure, and pressure from 0 to 1,450 psi was considered as low pressure.

Flow Meter of Oxygen Cylinders. The accuracy of oxygen cylinder flow meters was analyzed with a calibrated thermal mass flow meter (red-y compact GCM, Vögtlin, Switzerland) $(0-20 \mathrm{~L} / \mathrm{min}$, accuracy $1 \%$ of full scale or $\pm 0.2 \mathrm{~L} / \mathrm{min}$ ). Flow measurement with this thermal mass flow meter is independent of the temperature and atmospheric pressure. In each oxygen cylinder, different flows were evaluated in a random order $(2,4,6,9$, and $12 \mathrm{~L} / \mathrm{min}$ ). Randomization rate values were chosen with the random function of Microsoft Excel (Microsoft, Redmond, Washington). The delivered flow was quantified after 5 seconds at a steady state. Measurements were performed twice at each flow, and then the mean value was recorded. Flows of $2-4 \mathrm{~L} / \mathrm{min}$ and $6-12 \mathrm{~L} / \mathrm{min}$ were considered as low and high flow, respectively. 
Table 1. Source of Compressed Gas Cylinders

\begin{tabular}{lr}
\hline \hline Ambulance service & 36 \\
Emergency department & 88 \\
Fire brigade & 24 \\
Total & 148 \\
\hline
\end{tabular}

\section{Analysis}

Flows were expressed in standard units $(\mathrm{L} / \mathrm{min})$ and as percentage of required flow (\% required flow). For each flow, the minimum value, maximum value, range between both values, and interquartile range (IQR) were calculated. Mean values are expressed with their standard deviation for parametric data and median values with IQRs for non-parametric data. To evaluate the variability of oxygen cylinder flow meters, a coefficient of variation was calculated.

Single- and dual-stage groups were compared using an analysis of variance test followed by a Holm-Sidak method for parametric data. A Kruskall-Wallis test followed by Dunn's method or Mann-Whitney rank-sum test was used for non-parametric comparisons.

To check the precision of measurements for the calibrated thermal mass flow meter, a Friedman test was performed to analyze 3 random oxygen flow measurements $(1.5,6,9$, and $12 \mathrm{~L} / \mathrm{min})$ carried out during the phase of precision experiment (10 dual-stage at maximal pressure). The intraclass correlation coefficients were calculated to verify the repeatability of 3 successive measurements of oxygen flows; intraclass correlation coefficients values $>0.75$ were considered to reflect excellent repeatability.

An error analysis for each measurement was determined by the difference between the delivered and required flow divided by required flow.

\section{Results}

In all, 148 oxygen cylinders were analyzed (single- and dual-stage). Their provenance and distribution is shown in Table 1. The distribution of oxygen cylinder was: 53\% (no. $=78$ ) with a single-stage oxygen cylinder (Messer Cutting Systems, Groß-Umstadt, Germany) and 47\% $($ no. $=70)$ with a dual-stage oxygen cylinder (Air Liquide, Paris, France).

\section{Gas Pressure}

The residual pressure for both oxygen cylinders ranged from 73 to 2,900 psi. The median (25th and 75 th percentile) residual pressure of single-stage was 1,885 psi
Table 2. Compressed Gas Cylinder Distribution

\begin{tabular}{lcc}
\hline \hline \multicolumn{1}{c}{ Type } & Single-Stage OGC & Dual-Stage OGC \\
\hline No. $(\%)$ & $78(53)$ & $70(47)$ \\
Water volume, no. & $2 \mathrm{~L}, 74$ & $2 \mathrm{~L}, 14$ \\
& $10 \mathrm{~L}, 4$ & $5 \mathrm{~L}, 56$ \\
Median residual pressure* & 1,885 psi $(1,033-2,900)$ & 2,175 psi $(1,087-2,900)$ \\
Low pressure, no. $(\%)$ & $26(33)$ & $27(39)$ \\
High pressure, no. $(\%)$ & $52(67)$ & $43(61)$ \\
& & \\
$*$ Median residual pressure is expressed within the 25th and 75 th percentiles. \\
OGC $=$ oxygen gas cylinder & & \\
psi $=$ pounds per square inch & & \\
Low pressure $=0-1,450$ psi \\
High pressure $=1,451-2,900$ psi
\end{tabular}

(1,033-2,900 psi) and 2,176 psi (1,087-2,900 psi) for dual-stage $(P=.86)$.

For single-stage, 26 oxygen cylinders (33\%) were found to be pressurized at low pressure and 52 oxygen cylinders $(67 \%)$ at high pressure; for dual-stage, 27 oxygen cylinders $(39 \%)$ were pressurized at low pressure and 43 oxygen cylinder (61\%) at high pressure (Table 2).

\section{Flow}

For all the oxygen cylinders, the dispersion of the median value extended from $100 \%$ to $109 \%$ of the required flow for single-stage, and from $95 \%$ to $97 \%$ for dual-stage. Mainly for single-stage, the median value, range, and IQR decreased with increasing required flow (Table 3). Significant IQR differences $(P=.001)$ were observed between single- and dual-stage (Table 3). However, the gap between single- and dual-stage decreased with the increase of the required flow value. The median value of delivered flow was equal to or greater than the required flow for single-stage and below the required flow for dual-stage (Fig. 1 and Table 3).

For single-stage, but not for dual-stage, median value differences were observed between low and high residual pressure. Indeed, with single-stage, the median values decreased at the same flow when the oxygen cylinders were pressurized at low residual pressure (Fig. 2).

\section{Variability of the Measurements}

No statistical difference $(P=.61)$ was found with regard to the coefficient of variation between single- and dual-stage (Table 4). The intraclass correlation coefficient ranged from 0.97 to 0.99 , reflecting excellent reproducibility of measurements for the thermal mass flow meter (Table 5). The error of measurement decreased when the flow increased, particularly for single-stage (Table 6).

\section{Discussion}

This study evaluated the accuracy of oxygen flow delivered by oxygen cylinder flow meters. Two brands of 
Table 3. Distribution of Delivered Oxygen Flows*

\begin{tabular}{|c|c|c|c|c|c|c|c|c|c|c|}
\hline \multirow{2}{*}{$\begin{array}{c}\text { Required } \mathrm{O}_{2} \\
\text { Flow (L/min) }\end{array}$} & \multicolumn{5}{|c|}{ Single Stage } & \multicolumn{5}{|c|}{ Dual Stage } \\
\hline & 2 & 4 & 6 & 9 & 12 & 2 & 4 & 6 & 9 & 12 \\
\hline Max & $128 \%$ & $128 \%$ & $116 \%$ & $111 \%$ & $117 \%$ & $118 \%$ & $114 \%$ & $114 \%$ & $113 \%$ & $116 \%$ \\
\hline P75 & $120 \%$ & $113 \%$ & $104 \%$ & $103 \%$ & $104 \%$ & $98 \%$ & $100 \%$ & $100 \%$ & $98 \%$ & $99 \%$ \\
\hline Median & $109 \%$ & $104 \%$ & $100 \%$ & $100 \%$ & $100 \%$ & $95 \%$ & $96 \%$ & $97 \%$ & $95 \%$ & $95 \%$ \\
\hline $\mathrm{P} 25$ & $100 \%$ & $95 \%$ & $96 \%$ & $95 \%$ & $95 \%$ & $89 \%$ & $93 \%$ & $93 \%$ & $81 \%$ & $92 \%$ \\
\hline Min & $85 \%$ & $79 \%$ & $84 \%$ & $83 \%$ & $85 \%$ & $78 \%$ & $80 \%$ & $77 \%$ & $77 \%$ & $77 \%$ \\
\hline IQR & $20 \%$ & $18 \%$ & $9 \%$ & $8 \%$ & $9 \%$ & $9 \%$ & $8 \%$ & $8 \%$ & $7 \%$ & $7 \%$ \\
\hline Range & $43 \%$ & $49 \%$ & $32 \%$ & $28 \%$ & $32 \%$ & $40 \%$ & $34 \%$ & $38 \%$ & $36 \%$ & $39 \%$ \\
\hline
\end{tabular}

* Expressed as a percentage of the difference between the required and delivered oxygen flow between single- and dual-stage.

$\mathrm{IQR}=$ interquartile range

$\mathrm{P} 75=75$ th percentile

$\mathrm{P} 25=25$ th percentile
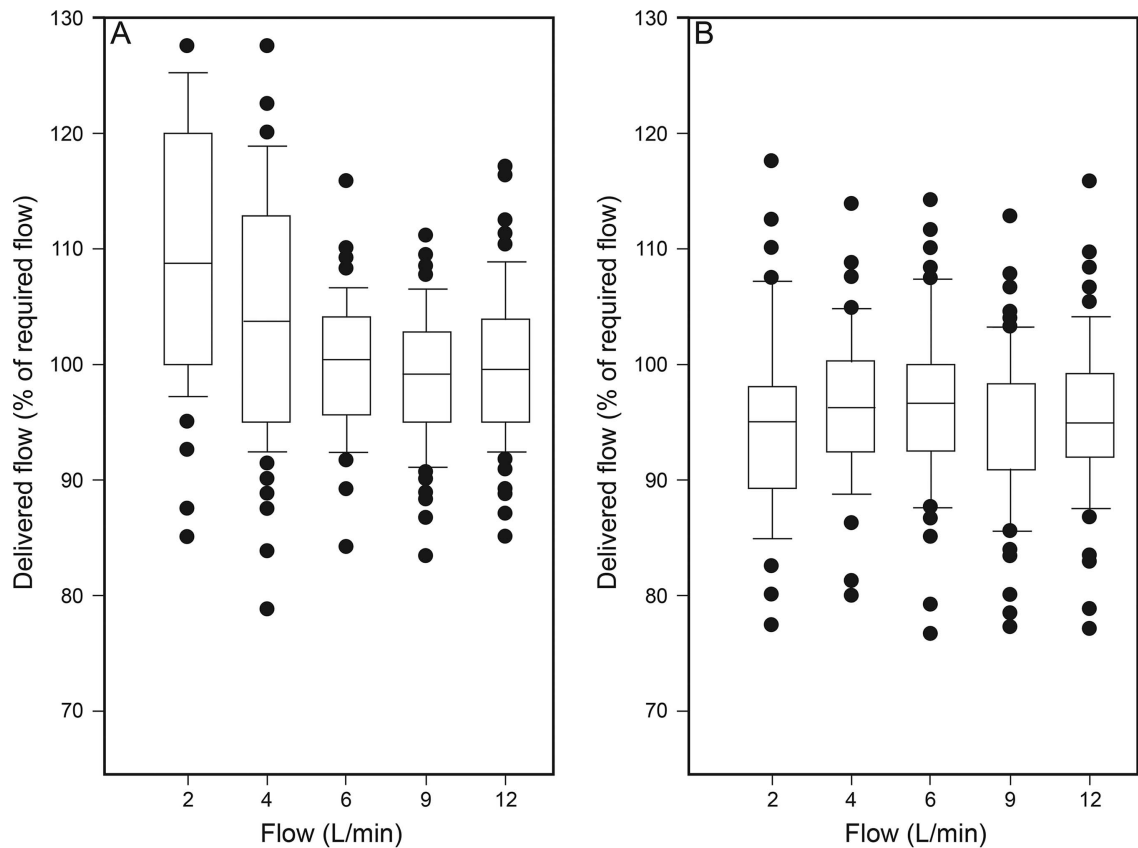

Fig. 1. Delivered flows were expressed as a percentage of required oxygen flow. Values obtained using 78 single-stage (A) and 70 dual-stage regulators (B) for oxygen flows from 2 to $12 \mathrm{~L} / \mathrm{min}$. Boxes illustrate the 25 th and 75 th percentiles, whiskers correspond to the 5th and 95th percentiles, and dots are outliers.

oxygen cylinder were evaluated. We found good accuracy of delivered oxygen flow for both types of oxygen cylinder analyzed. Slight differences in the flow between the brands were observed. While the difference between single- and dual-stage regulators was measurable, the difference was unlikely to be clinically important. On average, single-stage tended to deliver flow above required flow (mainly at low flow), while dual-stage delivered flow below required flow. The dispersion of the measurements was slightly greater with single-stage than dualstage. Independent of the pressure regulator system, delivered flows were, in general, close to required flow.
The error analysis showed that, when the required flow value increased, the percent error value decreased. This systematic error decreased with increasing flow, mainly for single-stage.

A similar study performed on a greater number of Thorpe tubes in hospitals (no. $=476$ ) showed median values of oxygen flow ranging from $91 \%$ to $110 \%$ of required flow. ${ }^{7}$ The dispersion of the measurements is greater with Thorpe tubes than with oxygen cylinder flow meters. With the single-stage, but not for dual-stage, median values of oxygen flow were lower with low gas pressure than with high gas pressure. This indicated that a decrease in 

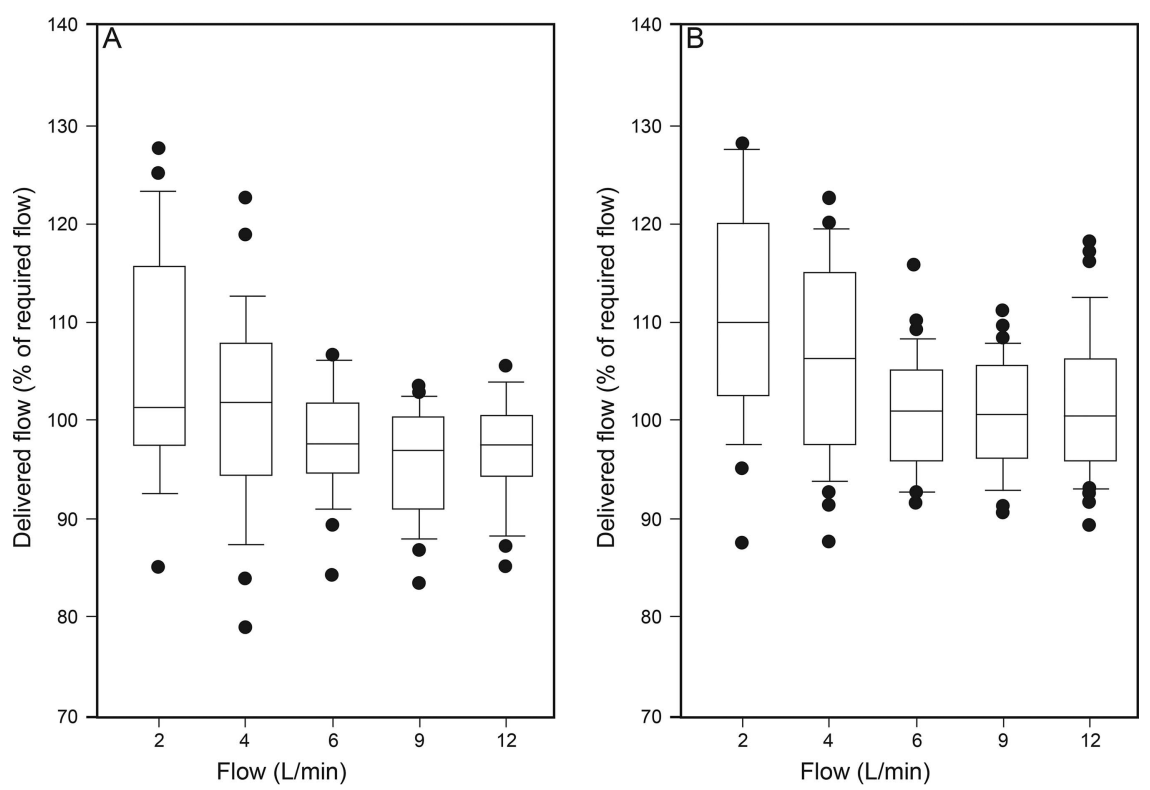

Fig. 2. Dispersion of the delivered flow differences for 78 single-stage oxygen cylinders between low pressure (A: ranged from 290 to $1,378 \mathrm{psi}$, no. $=26$ ) and high pressure (B: ranged from 1,450 to $2,900 \mathrm{psi}$, no. $=52$ ). Comparisons of low pressure and high pressure using the Friedman test are as follows: $2 \mathrm{~L} / \mathrm{min}, P=.041 ; 4 \mathrm{~L} / \mathrm{min}, P=.02 ; 6 \mathrm{~L} / \mathrm{min}, P=.02 ; 9 \mathrm{~L} / \mathrm{min}, P=.002$, and $12 \mathrm{~L} / \mathrm{min}, P=$ .004 .

Table 4. Mean of Delivered Flows and Coefficient of Variation Comparison Between Single- and Dual-Stage

\begin{tabular}{lrrrrr}
\hline \hline $\begin{array}{l}\text { Required } \\
\text { Flow }\end{array}$ & Mean $( \pm \mathrm{SD}), \mathrm{L} / \mathrm{min}$ & $P^{*}$ & \multicolumn{3}{c}{$\begin{array}{c}\text { Coefficient } \\
\text { of Variation } \\
P=.61^{* *}\end{array}$} \\
\hline $2 \mathrm{~L} / \mathrm{min}$ & $2.2( \pm 0.2)$ & $1.9( \pm 0.2)$ & $<.001$ & 0.11 & 0.09 \\
$4 \mathrm{~L} / \mathrm{min}$ & $4.2( \pm 0.4)$ & $3.9( \pm 0.3)$ & .003 & 0.10 & 0.07 \\
$6 \mathrm{~L} / \mathrm{min}$ & $6.0( \pm 0.3)$ & $5.8( \pm 0.4)$ & $<.001$ & 0.06 & 0.07 \\
$9 \mathrm{~L} / \mathrm{min}$ & $8.9( \pm 0.5)$ & $8.5( \pm 0.6)$ & $<.001$ & 0.06 & 0.07 \\
$12 \mathrm{~L} / \mathrm{min}$ & $12.0( \pm 0.8)$ & $11.5( \pm 0.9)$ & $<.001$ & 0.07 & 0.07
\end{tabular}

* Analysis of variance followed by Holm-Sidak method.

** Student's $t$-test.

pressure for single-stage had an impact on the delivered flow by decreasing this value. This difference can be explained by the automatic compensation for any drop in the supply pressure with a dual-stage, which allows the delivered flow to be kept constant. Moreover, dual-stage controlled gas pressures with more accuracy than single-stage because the pressure was gradually reduced through multistage regulators. ${ }^{5}$ Finally, dual-stage produced flows that were more constant than those from single-stage. Taking into consideration these issues, dual-stage had less variability than single-stage. These results are consistent with two previous papers that analyzed the accuracy of the flow of Thorpe tubes in a hospital. ${ }^{7,8}$ The conclusion of these studies was that the Thorpe tubes tested showed poor accuracy. On the other hand, our results revealed that oxygen cylinders have less variability of flow than
Table 5. Repeatability of Three Measurements of Required $\mathrm{O}_{2}$ Flow

\begin{tabular}{lccc}
\hline \hline & \multicolumn{3}{c}{ Flow Analyzer* } \\
\cline { 2 - 4 } Required Flow & Median & $25^{\text {th }}$ & $75^{\text {th }}$ \\
\hline $1.5 \mathrm{~L} / \mathrm{min}$ & & & \\
1 & 1.3 & 1.2 & 1.5 \\
2 & 1.3 & 1.2 & 1.5 \\
3 & 1.3 & 1.3 & 1.5 \\
$6 \mathrm{~L} /$ min & & & \\
1 & 5.8 & 5.5 & 6.1 \\
2 & 5.8 & 5.5 & 6.2 \\
3 & 5.9 & 5.6 & 6.2 \\
$9 \mathrm{~L} /$ min & & & 8.6 \\
1 & 8.4 & 8.1 & 8.6 \\
2 & 8.4 & 8.2 & 8.8 \\
3 & 8.4 & 8.2 & \\
12 L/min & & & 12.2 \\
1 & 11.1 & 10.7 & 12.2 \\
2 & 11.1 & 10.7 & \\
3 & 11.1 & 10.7 & \\
& & & \\
\hline Measurements were taken with 10 ocGs at full pressure. & & \\
$*$ Using the Friedman Test. & & & \\
\hline
\end{tabular}

Thorpe tubes, which means that the accuracy of oxygen cylinder flow meters is better than that of Thorpe tubes. Furthermore, the coefficient of variation in the study by Davidson et al (91 Thorpe tubes analyzed) was $0.6( \pm 0.5)$ and $0.11( \pm 0.01)$ for the study by Duprez et al (476 Thorpe tubes analyzed). ${ }^{7,8}$ In our study, the coefficient 


\section{Accuracy of Flow Delivered by Oxygen Cylinders}

Table 6. Error Analysis for Each Flow Between Single- and Dual-Stage

\begin{tabular}{llllll}
\hline \hline Single-stage & & & & & \\
$\quad$ & 2 & 4 & 6 & 9 & 12 \\
Required $\mathrm{O}_{2}, \mathrm{~L} / \mathrm{min}$ & 2.19 & 4.19 & 6 & 9 & 11.99 \\
$\quad \begin{array}{l}\text { Mean } \mathrm{O}_{2} \text { delivered, L/min } \\
\text { Percent error }\end{array}$ & $9.6 \%$ & $4.6 \%$ & $0 \%$ & $0 \%$ & $-0.1 \%$ \\
Dual-Stage & & & & & \\
$\quad$ Required $\mathrm{O}_{2}, \mathrm{~L} / \mathrm{min}$ & 2 & 4 & 6 & 9 & 12 \\
$\quad \begin{array}{l}\text { Mean } \mathrm{O}_{2} \text { delivered, L/min } \\
\text { Percent error }\end{array}$ & 1.89 & 3.85 & 5.79 & 8.52 & 11.46 \\
& $-5.5 \%$ & $-3.6 \%$ & $-3.5 \%$ & $-5.4 \%$ & $-4.5 \%$ \\
& & & & & \\
\hline
\end{tabular}

of variation was $0.08( \pm 0.02)$ for single-stage and 0.07 $( \pm 0.01)$ for dual-stage.

These differences of variability between oxygen cylinder flow meters and Thorpe tubes could be due to the red y compact (accuracy $\pm 1 \%$ for oxygen flow; range $0.5-20 \mathrm{~L} / \mathrm{min}$ ), which is a very accurate measuring instrument, whereas Davidson et al. used a Timeter RT-200 (Allied Healthcare Products, St. Louis, Missouri; accuracy $\pm 4 \%$ for oxygen flow; range $0.5-10 \mathrm{~L} / \mathrm{min}$ ). Furthermore, the flow meter of Thorpe tubes becomes inaccurate when exposed to static electricity or a magnetic field, secondary to a mechanical shock or a lack of verticality, and also to changes in atmospheric pressure or room temperature. ${ }^{5,9}$ These factors can lead to reading mistakes, which can explain why the accuracy of oxygen cylinder flow meters is higher than those of Thorpe tubes.

However, in 1996, Henderson et al ${ }^{10}$ studied the accuracy of oxygen flow through a nasal cannula in the operating room. They concluded that Thorpe tubes or oxygen cylinder flow meters provide accurate delivered oxygen flows. On the other hand, oxygen flows from the Ypiece of a circle system were lower than that of Thorpe tubes and oxygen cylinder flow meters, especially when the adjustable pressure limiting valve is open.

Despite this, patients who are oxygenated successively with different systems (oxygen cylinders with a flow meter vs Thorpe tubes) are unlikely to have a stable level of oxygenation because the accuracy of oxygen flow of these devices is different. However, oxygen is rarely titrated in prehospital care once hypoxia has been reversed. ${ }^{11}$ In addition, on arrival at the hospital, the oxygenation device is changed and could induce over- or under-oxygenation if $\mathrm{S}_{\mathrm{pO}_{2}}$ (or blood gas analysis) is not tested after this modification. As such, hyperoxia is a common finding upon hospital arrival in patients who received oxygen in prehospital care; hyperoxia is, in fact, just as common as hypoxia. ${ }^{11}$ It must be emphasized that the same situation described above could happen during intra-hospital transfers with the alternate use of Thorpe tubes and oxygen cylinders with flow meters.
Recent guidelines recommend using pulse oximetry more frequently to avoid hyperoxia. ${ }^{11}$ This should encourage the staff of emergency departments to be cautious and therefore to use pulse oximetry to determine the oxygen flow instead of the previous setting of flow after patient's arrival. They should also consider the difference of oxygen supply (and/or a modification of ventilatory pattern) as a cause of the perturbation before considering a change in patient's health status.

This study evaluated the accuracy of a large number of oxygen cylinder flow meters. Nonetheless, there are weaknesses in this study. Only 2 types of oxygen cylinder flow meters were studied, because in Belgium they were the only two readily available. Furthermore, during measurements, the ambient temperature was not taken into account, which could be a source of bias. Furthermore, the date of checking each oxygen cylinder flow meter was not known, because in Belgium, the law only requires oxygen cylinder flow meters to be checked every 5 years.

\section{Conclusions}

This study demonstrated that oxygen flow delivered by oxygen gas cylinders is accurate. Residual pressure influences the accuracy of oxygen flow, particularly with single-stage at low flow. Yet, oxygen cylinders with a flow meter have a lower variability than wall-mounted Thorpe tubes used in hospitals. Any change in oxygenation system (Thorpe tube vs oxygen cylinder with a flow meter, or between different oxygen cylinder flow meters) is a risky operation. Using $\mathrm{S}_{\mathrm{pO}_{2}}$ more frequently should reduce the risk of under- or over-oxygenation.

\section{ACKNOWLEDGMENTS}

The authors gratefully acknowledge the contributions of Frank Vantrimpont MD and Mr Christophe Fontaine at the Epicura Hospital Hornu, NT Michel Slingeneyer and NT Jacques Héraut at Ambroise Paré Hospital, Mons, Belgium, and Mr Francis Dupont of the emergency ambulance service Boussu for their assistance with the measurements for this study.

\section{REFERENCES}

1. Matthews A, Gavin C, O'Driscoll BR. Audit of pre-hospital oxygen therapy by North West Ambulance Service (NWAS) 1 year after publication of new JRCALC guidance for oxygen use. Thorax 2010; 65(Suppl):A174-A174.

2. O'Driscoll BR, Howard L, Bucknall C, Welham S, Davison A. British Thoracic Society emergency oxygen audits. Thorax 2011;66(8): 734-735.

3. Kane B, Decalmer S, O'Driscoll BR. Emergency oxygen therapy: from guideline to implementation. Breathe 2013;9(4):246-253.

4. Hale KE, Gavin C, O'Driscoll BR. Audit of oxygen use in emergency ambulances and in a hospital emergency department. Emerg Med J 2008;25(11):773-776. 


\section{Accuracy of Flow Delivered by Oxygen Cylinders}

5. Cairo JM, Pilbeam SP. Mosby's Respiratory Care Equipment. 7th edition. St. Louis: Mosby. 2013:84-85.

6. International Organization for Standardization. NF in ISO 15002: 2008-09. Flow-metering devices for connection to terminal units of medical gas pipeline systems. Available at: https://www.iso.org/en/standard/42057.html. Accessed June 10, 2017.

7. Duprez F, Barile M, Bonus T, Cuvelier G, Ollieuz S, Mashayekhi S, Legrand A. Accuracy of medical oxygen flow meters: a multicentric field study. Health 2014;06(15):1978-1983.
8. Davidson J, Gazzeta C, Torres LC, Jardim JR, Nascimento OA. Precision and accuracy of oxygen flow meters used at hospital settings. Respir Care 2012;57(7):1071-1075.

9. Hagelsten JO, Larsen OS. Inaccuracy of flowmeters caused by static electricity. Brit J Anaesth 1965;37(8):637-641.

10. Henderson CL, Rosen HD, Arney KL. Oxygen flow through nasal cannulae. Can J Anaesth 1996;43(6):636-639.

11. Branson RD, Johannigman JA. Pre-hospital oxygen therapy. Respir Care 2013;58(1):86-97. 